\title{
VIVÊNCIAS DE MULHERES NA MENOPAUSA: CONTRIBUTOS Ä COMPREENSÃO dO CUIDAR EM ENFERMAGEM
}

\author{
Elisa Santos \\ Instituto Politécnico de Viseu, Escola Superior de Saúde, Viseu, Portugal \\ elisaaugsantos@gmail.com \\ Emília Coutinho 2 \\ UICISA: E Instituto Politécnico de Viseu, Escola Superior de Saúde, Viseu, Portugal \\ ecoutinhoessv@gmail.com \\ Cláudia Chaves 3 \\ CIDEI, Instituto Politécnico de Viseu, Escola Superior de Saúde, Viseu, Portugal \\ claudiachaves21@gmail.com \\ Paula Nelas 4 \\ UICISA: E Instituto Politécnico de Viseu, Escola Superior de Saúde, Viseu, Portugal \\ pnelas@gmail.com
}

Recepción Artículo: 22 mayo 2021

Admisión Evaluación: 22 mayo 2021

Informe Evaluador 1: 23 mayo 2021

Informe Evaluador 2: 28 mayo 2021

Aprobación Publicación: 01 junio 2021

\section{RESUMO}

A menopausa é um período da vida da mulher que necessita de toda a atenção dos profissionais de saúde, e particularmente do enfermeiro Especialista em Enfermagem de Saúde Materna e Obstétrica. 0 objetivo deste estudo é compreender as vivências da mulher no período da menopausa, o significado atribuído pela mulher às intervenções de enfermagem desenvolvidas pelo enfermeiro Especialista em Enfermagem de Saúde Materna e Obstétrica durante o período da menopausa e pós-menopausa. Foi realizado um estudo qualitativo, com recurso ao método fenomenológico-hermenêutico, tendo como instrumento de recolha de dados a entrevista fenomenológica. As 13 participantes recorreram às consultas de um Hospital de Dia Ginecológico e do Serviço de Ginecologia de um Hospital Central da Região Centro de Portugal. A análise qualitativa de dados foi apoiada pelo Nvivo12. Da análise do verbatim das entrevistas, emergiram cinco categorias: "Significado atribuído à menopausa", sendo as subcategorias mais destacadas "Experiência positiva", "Não ter sentido alterações com a menopausa" e "Uma fase má/desconfortável"; "Alterações sentidas durante a menopausa e pós menopausa", evidenciando-se mais a subcategoria "Alterações orgânicas", "nomeadamente as "Ondas de calor"; "Estratégias utilizadas face aos constrangimentos", onde as subcategorias mais destacadas foram "Procurar ajuda profissional" e "Adotar uma atitude de resiliência"; "Expectativas quanto aos cuidados de enfermagem", com a subcategoria mais referida "Expetativas corresponderam ao esperado"; "Cuidados de enfermagem recebidos na menopausa", sobressaindo as subcategorias "Ajuda", "Esclarecimento de dúvidas" e "Capacitação da mulher para vivenciar a menopausa". Parte das mulheres encara- 


\section{VIVÊNCIAS DE MULHERES NA MENOPAUSA: CONTRIBUTOS À COMPREENSÃO DO CUIDAR EM ENFERMAGEM}

ram a menopausa de forma positiva. Para outras, demarcou-se como uma fase de desconfortos, com alterações das condições vaginais, emocionais, orgânicas e alterações da atividade sexual. As mulheres consideram, sobretudo, que os cuidados de enfermagem corresponderam às suas expetativas, pois houve esclarecimento de dúvidas e foram capacitadas para vivenciar a menopausa de forma positiva e saudável.

Palavras-chave: cuidar em enfermagem; mulheres; menopausa

\section{ABSTRACT}

Experiences of women in menopause: contributions to understanding nursing care. Tradução em Inglês.Menopause is a period in a woman's life that needs all the attention of health professionals, especially the nurse specialist in Maternal and Obstetric Health Nursing. Thus, the objective was to understand the experiences of women during the menopause period, the meaning attributed by the woman to the nursing interventions developed by the Nurse Specialist in Maternal and Obstetric Health Nursing during the menopause and post-menopause period. A qualitative study was carried out, using the phenomenological-hermeneutic method, using the phenomenological interview as an instrument for data collection. The 13 participants resorted to consultations at a Gynecological Day Hospital and the Gynecology Service of a Central Hospital in the Central Region of Portugal. Qualitative data analysis was supported by Nvivo12. From the verbatim analysis of the interviews, five categories emerged: "Meaning attributed to menopause", with the most prominent subcategories being "Positive experience", "Not having experienced changes with menopause" and "A bad / uncomfortable phase"; "Changes felt during menopause and post menopause", with more emphasis on the subcategory "Organic changes", "namely the" Heat waves "; "Strategies used in the face of constraints", where the most prominent subcategories were "Seeking professional help" and "Adopting an attitude of resilience"; "Expectations regarding nursing care", with the most referred subcategory "Expectations corresponded to what was expected"; "Nursing care received during menopause", with emphasis on the subcategories "Help", "Clarification of doubts" and "Training of women to experience menopause". Some women viewed menopause positively. For others, it was marked as a phase of discomfort, with changes in vaginal, emotional, organic conditions and changes in sexual activity. Women consider, above all, that the nursing care corresponded to their expectations, as doubts were clarified and they were trained to experience menopause in a positive and healthy way.

Keywords: nursing care; women; menopause

\section{INTRODUÇÃO}

A palavra "menopausa" deriva das palavras gregas emmenopausis / emmenopausi, que significa "fim do ciclo dos meses". É composta a partir da união de dois termos gregos: $m$ n, que pode ser traduzido como "mês" ou "luas" (contagem lunar que delimita o período de um mês); e a palavra pausis, que significa "cessação", "pausa" ou mesmo "término". Na língua portuguesa, este termo surgiu a partir do francês ménopause, que deriva etimologicamente do termo médico latino menopausis (Dicionário Etimológico, s.d.). Geralmente é identificada pela ausência de menstruação por 12 meses. É muitas vezes considerada "um evento significativo na vida de uma mulher e é acompanhada por uma série de mudanças biológicas e psicossociais, tendo estas de se adaptar a uma nova etapa da sua vida" (Samarasiri, Rajanayake, Wattegedara, Kodituwakku, Senadheera \&, Silva, 2018, p. 43).

A forma como a transição para a menopausa é vivenciada pelas mulheres pode depender das suas normas culturais, aprendizagem social e conhecimento pessoal sobre a menopausa. A experiência da menopausa é culturalmente determinada e, portanto, as mulheres têm ideias preconcebidas sobre esta (Dillaway, 2020, p. 258). De acordo com a autora, muitas mulheres na menopausa referem a mudança da feminilidade, estarem a viver num corpo em completa transformação, alteração a nível do relacionamento com os outros e mudanças psicológicas.

Por conseguinte, é fundamental conhecer as vivências de mulheres durante a menopausa, como forma de enquadrar uma prática profissional que responda às necessidades de cada mulher de forma individualizada, perspetivando-se um cuidar holístico. Neste sentido, realça-se que o cuidado de enfermagem se configura como um fenómeno intencional, fundamental à vida, que acontece na relação entre os seres humanos que interagem através 
de atitudes que compreendem perceção, zelo, solidariedade, assertividade e empatia. Significa um "saber-fazer" que se fundamenta "na ciência, na arte e na ética, direcionado às necessidades da pessoa, da família e da comunidade" (Vale \& Pagliuca, 2011, p. 22).

Os sintomas que surgem durante a menopausa variam entre as mulheres, sendo alguns toleráveis e outros mais complexos, o que leva a que algumas mulheres tenham de recorrer à terapêutica hormonal de substituição (The North American Menopause Society, 2014, s.p.). A menopausa, por norma, interfere no bem-estar das mulheres, devido às alterações fisiológicas. Ao longo da peri-menopausa, os níveis de estrogénios progressivamente vão baixando e, por consequência, resultam na ocorrência de sintomas vasomotores, psicológicos e de atrofia do trato urogenital, desregulando o centro termorregulador hipotalâmico, que, por sua vez, tem interferência na qualidade de vida da mulher. No que se refere aos sintomas vasomotores, que são controlados pelo hipotálamo, destacam-se as ondas de calor/afrontamentos, a sudorese, as insónias, as cefaleias, a irritabilidade e a fadiga (Kaunitz \& Manson, 2015, pp. 395-396). Destacam-se também as mudanças psicológicas, nomeadamente, as alterações de humor, a ansiedade e a depressão (Probo, Soares, Silva, \& Cabral, 2016, pp. 245-246).

\section{OBJETIVOS DE PESQUISA}

0 objetivo geral do estudo é compreender o significado atribuído pela mulher à vivência de acontecimentos ocorridos durante a menopausa e pós-menopausa como a atrofia vulvovaginal/síndrome geniturinária e aos cuidados desenvolvidos pelos enfermeiros Especialistas em Enfermagem de Saúde Materna e Obstétrica durante 0 processo. Como objetivos específicos delinearam-se os seguintes: (i) Compreender as vivências da mulher no período da menopausa; (ii) Compreender o significado atribuído pela mulher às intervenções de Enfermagem de Saúde Materna e Obstétrica durante o período da menopausa e pós-menopausa.

\section{PARTICIPANTES}

As participantes deste estudo são 13 mulheres, utentes do Hospital Dia Ginecológico e do Serviço de Ginecologia de um Hospital Central da Região Centro de Portugal. A maioria possui idade igual ou superior a 55 anos $(61,54 \%)$, casadas (92,31\%), com escolaridade até ao $9 .{ }^{\circ}$ ano (46,15\%), possuindo uma profissão não qualificada (38,46\%). Em 61,54\% das mulheres, 0 início da menopausa ocorreu em idade $\geq 50$ anos.

\section{METODOLOGIA}

0 presente estudo é de natureza descritiva, exploratória e qualitativa com enfoque fenomenológico-hermenêutico. A pesquisa fenomenológica objetiva a distinção entre a aparência e a essência, de tal forma que o investigador tenta compreender em que constitui o fenómeno e a experiência vivida em questão (van Manen, 2017, pp. 775-776). Trata-se de uma pesquisa qualitativa, método utilizado quando não se sabe muito sobre uma determinada situação. A pesquisa qualitativa pode ajudar a clarificar um fenómeno que mais tarde pode ser testado por métodos quantitativos (Bogdan \& Biklen, 2007, p. 133). A fenomenologia procura explicar significados pessoais e, entender a perceção sobre os relacionamentos, expectativas, atitudes e crenças, bem como o significado com sentido resultante da experiência vivida, ou seja, a fenomenologia configura-se para Van Manen (1997, p. 24) como um método que permite interpretar. Neste sentido, Greenblum, Meredeth, Rowe, Neff e Greenblum (2013, p. 23) entendem que a riqueza, as variações, os pontos em comum ou as diferenças da transição da menopausa, vivenciada pelas mulheres, podem ser documentadas através de uma abordagem fenomenológica. Assim, neste estudo, o método de colheita de dados que melhor se adequou aos objetivos foi a entrevista fenomenológica, elaborada para 0 efeito, em que as questões centrais foram: 0 que significa para si a experiência vivida na menopausa e pós-menopausa? 0 que significou para si ser cuidada pelo enfermeiro especialista em enfermagem de saúde materna e obstétrica? É de referir que a recolha de dados decorreu entre 15 de novembro a 31 de dezembro de 2019, no Serviço de Ginecologia do Centro Hospitalar onde 0 estudo decorreu, com a duração de cada entrevista entre 20 a 30 minutos, tendo estas sido gravadas em suporte áudio e, posteriormente, transcritas. Foi efe- 
tuado e obtido um pedido de autorização para realização das entrevistas à Comissão de Ética do Centro Hospitalar onde 0 estudo foi realizado e respeitados todos os princípios éticos inerentes a este tipo de investigação.

\section{RESULTADOS ALCANÇADOS}

Os dados recolhidos através das entrevistas encontram-se agrupados em cinco categorias, designadamente: "Significado atribuído à menopausa", "Alterações sentidas durante a menopausa e pós menopausa", "Estratégias utilizadas face aos constrangimentos", "Expectativas quanto aos cuidados de enfermagem" e "Cuidados de enfermagem recebidos na menopausa". Cada categoria ficou constituída por subcategorias que reúnem as citações mais expressivas recolhidas do testemunho das participantes entrevistadas. As categorias e subcategorias são apresentadas tendo em conta o número de mulheres que se referem ao tema (n) e a frequência com que o fazem (Ur).

\section{Significado atribuído à menopausa}

A categoria "Significado atribuído à menopausa" é constituída por seis subcategorias, nomeadamente: "Não ter sentido alterações com a menopausa", "Experiência positiva", "Uma fase má/desconfortável", "Ter mais complicações de saúde na menopausa", "Sintomas ligeiros" e "Antecipar o tratamento de compensação". As subcategorias mais referenciada pelas informantes foram "Não ter sentido alterações com a menopausa" referenciada por quatro mulheres com oito unidades de registo, "Experiência positiva" contando com seis unidades de registo referidas por cinco mulheres "Uma fase má/desconfortável" relatada por quatro mulheres e com quatro unidades de registo. Também é de realçar o testemunho de duas mulheres que referem terem mais complicações de saúde na menopausa (cf. Tabela 1).

Tabela 1. Significado atribuído à menopausa

\begin{tabular}{|c|c|c|}
\hline Significado atribuído à menopausa & $\mathbf{N}$ & $\mathbf{U r}$ \\
\hline Não ter sentido alterações com a menopausa & 4 & 8 \\
\hline Experiência positiva & 5 & 6 \\
\hline Uma fase má/desconfortável & 4 & 4 \\
\hline $\begin{array}{l}\text { Ter mais complicações de saúde na } \\
\text { menopausa }\end{array}$ & 2 & 2 \\
\hline Sintomas ligeiros & 2 & 2 \\
\hline Antecipar o tratamento de compensação & 1 & 1 \\
\hline
\end{tabular}

Apresentam-se algumas unidades de registo expressivas desta categoria

"Pois lá está, tive uma experiência positiva [a vivência da menopausa]" (E4).

"A minha menopausa foi uma santa menopausa, tanto a nível físico de dores, como a nível da sexualidade. Não senti problemas nenhuns" (E8).

"Comecei a ter mais complicações de saúde após a menopausa" (E6).

"Às vezes sinto coisas leves comparadas com as queixas de outras pessoas" (E5).

\section{Alterações sentidas durante a menopausa e pós-menopausa}

A categoria "Alterações sentidas durante a menopausa e pós-menopausa" é constituída por quatro subcategorias, nomeadamente: "Alterações orgânicas", "Alterações emocionais", "Alterações das condições vaginais" e "Alteração da atividade sexual" (cf. Tabela 2). Nas "Alterações orgânicas", as mais referenciadas foram as "ondas de calor" (n=31 unidades de registo). A subcategoria "Alterações emocionais" é constituída por cinco sub-especificações, nomeadamente: irritabilidade com 8 unidades de registo, labilidade emocional com 7 referenciações; sentir 0 peso da idade referenciada 4 vezes; 0 mau estar apontado por duas participantes e a timidez referenciada apenas por uma mulher. As alterações das condições vaginais identificadas pelas informantes são: secura vaginal, referida por 
cinco das treze participantes; o prurido vaginal foi apontado por três participantes; 0 ardor vaginal referenciado por duas participantes e 0 desconforto vaginal enunciado por duas participantes. No que se refere às "Alterações da atividade sexual", a mesma surge com 3 unidades de registo e a dispareunia foi referenciada 5 vezes.

Tabela 2. Alterações sentidas durante a menopausa e pós-menopausa

\begin{tabular}{lcc} 
Alterações sentidas durante a menopausa & N & Ur \\
\hline e pós-menopausa & & \\
\hline Alterações orgânicas & 11 & 31 \\
Ondas de calor & 4 & 5 \\
A cessação da menstruação & 1 & 3 \\
Dores ósseas & 2 & 2 \\
Enxaqueca & 1 & 2 \\
Distinção abdominal & 1 & 1 \\
Hemorragia & & \\
Alterações emocionais & 4 & 8 \\
Irritabilidade & 4 & 7 \\
Labilidade emocional & 2 & 4 \\
Sentir o peso da idade & 2 & 2 \\
Mau estar & 1 & 1 \\
Timidez & & \\
Alterações das condições vaginais & 3 & 6 \\
Prurido vaginal & 5 & 5 \\
Secura vaginal & 2 & 3 \\
Desconforto vaginal & 2 & 2 \\
Ardor vaginal & 3 & 3 \\
Alteração da atividade sexual & 5 & 5 \\
Dispareunia &
\end{tabular}

De seguida apresentam-se alguns testemunhos

"Tive secura vaginal" (E7).

"Tinha comichão" (E78).

"De vez em quando tenho ardor vaginal" (E10).

"Sentia muitas oscilações de humor" (E2).

"Mais irritada" (E6).

"Bocadinhos de mau estar" (E12).

"Pensava que estava a ficar velha" (E11).

"Diferenças de temperatura abruptas" (E1).

"Só senti a perda do periodo" (E5).

"Cheguei a ter fortes enxaquecas" (E13).

"[senti] a barriga muito inchada [ao longo da menopausa e pós-menopausa]" (E2).

"Esses problemas que tenho de ossos" (E3).

"Tive problemas de bexiga" (E3). 


\section{Estratégias utilizadas face aos constrangimentos}

A categoria "Estratégias utilizadas face aos constrangimentos" é constituída por três subcategorias, nomeadamente: "Procurar ajuda profissional", "Adotar uma atitude de resiliência" e "Recorrer a saberes populares". "Procurar ajuda profissional" foi a mais referenciada ( $n=17$ unidades de registo), seguindo-se "Adotar uma atitude de resiliência", com 9 referências. Na subcategoria "Recorrer a saberes populares", as sub-especificações mais referenciadas pelas participantes foram: evitar fármacos, referenciada 3 vezes; abanar-se com um leque referenciada 2 vezes e molhar os pés em água fria, com 2 referenciações (cf. Tabela 3).

Tabela 3. Estratégias utilizadas face aos constrangimentos

\begin{tabular}{lcc}
$\begin{array}{l}\text { Estratégias utilizadas face aos } \\
\text { constrangimentos }\end{array}$ & N & Ur \\
\hline Procurar ajuda profissional & 9 & 17 \\
Adotar uma atitude de resiliência & 6 & 9 \\
$\quad$ Utilizar lubrificantes & 3 & 6 \\
$\quad \begin{array}{l}\text { Recorrer a terapia hormonal } \\
\text { Recorrer a saberes populares } \\
\text { Evitar fármacos }\end{array}$ & 3 & 5 \\
Abanar-se com um leque & 2 & 3 \\
$\quad$ Molhar os pés em água fria & 2 & 2 \\
Beber água & 1 & 2 \\
Trocar a roupa transpirada por roupa & 1 & 1 \\
seca $\quad 1$ & 1 \\
$\quad$ Dormir com os pés fora da roupa & 1 & 1 \\
Lavar a vagina com tisanas & 1 & 1 \\
$\quad$ Respirar profundamente & 1 & 1 \\
Evitar o ambiente ruidoso & 1 & 1 \\
Ocupar-se com atividades de vida & 1 & 1 \\
diária & & \\
\hline
\end{tabular}

Apresentam-se algumas expressões das participantes

"Não tem de se recorrer sempre a fármacos "(E5).

"Eram os leques que eu tinha que utilizar [para diminuir o calor]" (E12).

"Medicamentos não tomo e quando tenho muito calor, vou beber água e passame logo" (E10).

"Lavava os pés com água fria e voltava-me a meter na cama" (E13).

"Como a roupa ficava toda molhada por causa dos calores também tinha de mudar de pijama" (E13).

"Eu mesma fazia uma espécie de uma tisana, quer dizer um chá, com eucalipto ou alecrim, fazia ferver água e deixava em efusão para fazer as lavagens" (E7). "Tentava respirar fundo [quando tinha variações de humor] para não afetar quem me rodeava" (E2).

"Só dizia, não façam barulho que não posso ouvir ninguém" (E12).

"Ocupando-me com as minhas atividades de vida diária" (E2). 


\section{Expectativas quanto aos cuidados de enfermagem}

A categoria "Expectativas quanto aos cuidados de enfermagem" é constituída por sete subcategorias: "Expetativas corresponderam ao esperado"; "Sem expectativas"; "Ser informada sobre 0 que a poderá ajudar na menopausa"; "Superação das expectativas"; "Ser capacitada para a tomada de decisões", "Que aliviassem o prurido" e "Receber atenção e apoio", sendo as "Expetativas corresponderam ao esperado" a mais referida pelas participantes, com cinco referenciações e cinco unidades de registo (cf. Tabela 4).

Tabela 4. Expectativas quanto aos cuidados de enfermagem

\begin{tabular}{lccc}
$\begin{array}{l}\text { Expectativas quanto aos cuidados de } \\
\text { enfermagem }\end{array}$ & N & Ur \\
\hline Expetativas corresponderam ao esperado & 5 & 5 \\
Sem expectativas & 3 & 5 \\
Ser informada sobre o que a poderá ajudar na & 2 & 3 \\
menopausa & 2 & 3 \\
Superação das expectativas & 1 & 2 \\
Ser capacitada para a tomada de decisões & 1 & 1 \\
Que aliviassem o prurido & 1 & 1 \\
Receber atenção e apoio & \\
\hline
\end{tabular}

Seguidamente apresentam-se algumas expressões das participantes

"A expectativa foi ao encontro do que pensava que ia surgir" (E6).

"Não tinha [expectativas quanto aos cuidados de enfermagem na menopausa]"

(E1).

"As enfermeiras têm conhecimentos que nos são muito úteis" (E9).

"Que me desse uma medicação que me ajude nesta situação, comichão" (E3).

"O apoio psicológico [por parte dos enfermeiros] é muito melhor [do que os fármacos], para não sermos negativas" (E5).

"A gente por vezes vem aqui e não precisa de um comprimido, mas de 5 minutos de conversa que a gente sinta que está a ser ouvida e que o outro [a enfermeira] nos dá um apoio e nos dá atenção, o que nos vai fazer bem" (E5).

\section{Cuidados de enfermagem recebidos na menopausa}

A categoria "Cuidados de enfermagem recebidos na menopausa" é constituída por treze subcategorias, sendo as mesmas: "Esclarecimento de dúvidas", "Ajuda"; "Capacitação da mulher para vivenciar a menopausa"; "Orientação e aconselhamento", "Sem necessidade de cuidados de enfermagem"; "Escuta ativa", "Compreensão empática"; "Cuidados humanizados"; "Apoio psicológico"; "Alívio da dor e desconforto"; "Promoção do autocuidado e bem-estar"; "Ensinar"; "Sem necessidade de fazer terapêutica". A subcategoria "Esclarecimento de dúvidas", "Ajuda", "Capacitação da mulher para vivenciar a menopausa" e "Orientação e aconselhamento" foram as que reuniram mais unidades de registo (cf. tabela 5). 


\begin{tabular}{|c|c|c|}
\hline $\begin{array}{l}\text { Cuidados de enfermagem recebidos na } \\
\text { menopausa }\end{array}$ & $\mathbf{N}$ & $\mathbf{U} \mathbf{r}$ \\
\hline Esclarecimento de dúvidas & 7 & 17 \\
\hline Ajuda & 9 & 15 \\
\hline $\begin{array}{l}\text { Capacitação da mulher para vivenciar a } \\
\text { menopausa }\end{array}$ & 7 & 14 \\
\hline Orientação e aconselhamento & 4 & 11 \\
\hline Sem necessidade de cuidados de enfermagem & 5 & 10 \\
\hline Escuta ativa & 5 & 9 \\
\hline Compreensão empática & 6 & 7 \\
\hline Cuidados humanizados & 5 & 7 \\
\hline Apoio psicológico & 5 & 5 \\
\hline Alívio da dor e desconforto & 3 & 4 \\
\hline Promoção do autocuidado e bem-estar & 3 & 4 \\
\hline Ensinar & 1 & 1 \\
\hline Sem necessidade de fazer terapêutica & 1 & 1 \\
\hline
\end{tabular}

Apresentam-se algumas expressões das participantes

"A enfermeira ajudou-me muito" (E4).

"O mais importante é que a enfermeira me explicou tudo" (E2).

"[a enfermeira foi importante] para eu ultrapassar os sintomas" (E4).

"[a enfermeira] foi compreensiva [com o que eu estava a viver]" (E4).

"Não falei com nenhum enfermeiro" (E3).

"[A enfermeira] deu-me apoio" (E6).

"[A enfermeira] foi muito humana comigo, [a enfermeira] foi uma amiga, se

assim posso dizer" (E2).

"a enfermeira sempre me ouviu" (E2).

"[a enfermeira] dava-me orientações " (E4).

"A senhora enfermeira ajudou-me a ter bem-estar perante as alterações

fisiológicas que eu sentia" (E2).

\section{DISCUSSÃO}

De seguida discutem-se os resultados com os de outros autores, apresentando-se as categorias pela ordem em que foram apresentadas nos resultados. Na categoria "Significado atribuído à menopausa", emergiram as subcategorias "Não ter sentido alterações com a menopausa", "Experiência positiva", "Uma fase má/desconfortável", "Ter mais complicações de saúde na menopausa", "Sintomas ligeiros" e "Antecipar o tratamento de compensação". No seu estudo, Silva e Mamede (2017, pp.3-5), constaram que o significado atribuído pelas mulheres à menopausa foi sobretudo ser a transição para o envelhecimento e o surgimento de doenças, uma fase marcada por várias transformações físicas e psicológicas, classificando esta como uma fase negativa, o que está em conformidade com o depoimento de algumas das mulheres do presente estudo. No entanto, a literatura mostra que a experiência subjetiva das mulheres na menopausa nem sempre é puramente negativa. As mulheres podem ter muitas respostas positivas ou neutras face aos sintomas (Dillaway, 2020, pp. 257-258). De acordo com a mesma autora, as mulheres podem ter uma variedade de reações, atitudes e experiências face aos sintomas da menopausa, 0 que influencia o significado que atribuem à menopausa, ou seja, reações negativas, positivas, neutras e algumas até confusas. A mesma autora salienta que um sinal ou sintoma pode trazer múltiplas reações, atitudes e experiências. Por um lado, passar do período menstrual pode ser libertador, mas, por outro lado, pode induzir 
ansiedade. Da mesma forma, ter ondas de calor pode ser constrangedor em público pela primeira vez, mas algumas mulheres aprendem rapidamente como lidar com esta situação e ajustam a dieta e 0 vestuário a fim de moderar esse sintoma físico. Nestes caso, uma reação inicial a um sintoma corporal pode ser positiva ou negativa, mas as reações, atitudes e experiências podem ajustar-se com o tempo (Dillaway, 2020, p. 258). De igual modo, Gault (2015) refere que a menopausa não é necessariamente uma experiência negativa para todas as mulheres que se encontram nesta fase da sua vida, uma vez que há mulheres que experienciam a menopausa sem nenhum sintoma negativo. Neste estudo, na categoria "Alterações sentidas durante a menopausa e pós-menopausa", a subcategoria mais referenciada foi "Alterações orgânicas", onde sobressaíram as "ondas de calor". Na subcategoria "Alterações emocionais", a irritabilidade e a labilidade emocional foram as mais evidentes. As alterações das condições vaginais identificadas pelas informantes foram: secura vaginal, o prurido vaginal, 0 ardor vaginal e 0 desconforto vaginal. Houve também mulheres a referenciarem as "Alterações da atividade sexual". Estes resultados corroboram a literatura, uma vez que 0 impacto dos sintomas da menopausa pode variar de pessoa para pessoa, com algumas mulheres a experienciarem sintomas que afetam a sua condição física e 0 desempenho social, bem como a sua qualidade de vida (Waheed, Khanum, Ejaz, Butt \& Randhawa, 2016, pp. 1-2; (Samarasiri, Rajanayake, Wattegedara, Kodituwakku, Senadheera \& Silva, 2017, p. 43). No estudo de Andrade, Fernandes, Dias, Laurindo e Vieira (2019, p. 75-76), as mulheres estudadas apresentaram sintomatologia severa durante a menopausa, desde as alterações vaginais a alterações emocionais, com sintomas somato-vegetativos e psicológicos, mas representando estes uma sintomatologia moderada. Em pesquisas de coorte em populações ocidentais, ficou demonstrado que entre $45 \%$ a $63 \%$ das mulheres na pós-menopausa relataram que vivenciaram sintomas vulvovaginais, como a secura e 0 ardor vaginal, tendo também vivenciado outros sintomas como a irritação vaginal, a sensação de prurido, a sensibilidade vaginal (Nappi \& Kokot-Kierepa, 2010, p.233; Kingsberg, Wysocki, Magnus et al., 2013, p. 1790). De igual modo, no estudo de Chae, Choi, Cho, Cho, Lee, Lee et al., (2014, p.7), $49 \%$ das mulheres tinham experimentado a secura e 0 ardor vaginal. Resultados estes corroborados no presente estudo. Silva e Mamede (2017, p. 7) salientam que as mulheres na menopausa e pós-menopausa "necessitam de uma atenção mais qualificada que transcenda os aspetos biológicos da assistência à saúde, favorecendo um cuidado integral e mais próximo das suas necessidades e que contemple as suas singularidades" (p. 7).

No estudo de Samarasiri et al. (2017, p. 56), as mulheres revelaram dificuldades na tomada de decisões, esquecimento, irritação, alteração de humor e frustração, sendo estas as alterações cognitivas emocionais sentidas pelas mesmas. Neste estudo, as mulheres relataram ter dificuldade na tomada de decisões no seu dia a dia e na vida profissional. No entanto, algumas participantes revelaram que a menopausa não constituiu um problema para as suas atividades diárias. Beck, Brewis \& Davies (2019) referem que as alterações psicológicas resultantes da menopausa podem levar à frustração, ansiedade, irritabilidade, alterações de humor e depressão, o que muitas vezes tem um impacto nas relações pessoais e na qualidade de vida das mulheres. A categoria "Estratégias utilizadas face aos constrangimentos" é constituída por três subcategorias: "Procurar ajuda profissional", "Adotar uma atitude de resiliência" e "Recorrer a saberes populares". "Procurar ajuda profissional" foi a mais referenciada, seguindo-se "Adotar uma atitude de resiliência". Na subcategoria "Recorrer a saberes populares", as subespecificações mais referenciadas pelas participantes foram: evitar fármacos, abanar-se com um leque e molhar os pés em água fria. A propósito das estratégias utilizadas pelas mulheres na menopausa, Yazdkhasti, Simbar e Abdi (2015, pp. 3-4) referem que as mulheres devem ter acesso a mais informações sobre as suas necessidades físicas e psicossociais. Assim, o empowerment por parte dos profissionais de saúde durante a menopausa pode contribuir para melhorar a perceção deste estadio e contribuir para um melhor enfrentamento dos sintomas vivenciados, potenciando o autocuidado. É essencial aumentar a consciencialização e a adaptação das mulheres à menopausa, através de programas de empowerment. No seu estudo, os mesmos autores constaram que a maioria das intervenções por parte dos profissionais de saúde, com destaque para os enfermeiros, para as mulheres na menopausa concentrou-se em intervenções educacionais, promoção da atividade física/exercício, dieta saudável, controlo do stresse, adoção de comportamentos saudáveis, prevenção de doenças, como a osteoporose, bem como apoio psicológico para que as mulheres tenham mais resiliência. A categoria "Expectativas quanto aos cui- 
dados de enfermagem" teve como subcategoria mais referenciada as "Expetativas corresponderam ao esperado" a mais referida pelas participantes. No estudo de Pedras (2017), as mulheres relataram 0 apoio médico, tendo raramente mencionado o apoio do enfermeiro, o que difere do presente estudo, uma vez que a maioria das mulheres estudadas referiu que os cuidados de enfermagem corresponderam às suas expectativas. De igual modo, no estudo de Pedras (2017), a maioria das mulheres referiu que a menopausa se configurou como um processo de transição que ocorreu pior do que esperavam e, segundo as mesmas, o enfermeiro deveria informá-las e esclarecê-las acerca do assunto, aconselhando-as e orientando-as, passando também a sua ação pela desmistificação de mitos e crenças associados à menopausa, bem como deveriam, nos cuidados prestados, apoiá-las mais emocionalmente, e fazer uma vigilância do seu estado de saúde na consulta de enfermagem e através de rastreios. Neste sentido, importa referir que, particularmente os enfermeiros Especialistas em Saúde Materna, Obstetrícia e Ginecologia, devem ajudar as mulheres na mulher de modo a poderem prestar cuidados que possam ir ao encontro das suas necessidades fisiológicas, reprodutivas e psicológicas (Gass, Cochrane Lars et al., 2011, p. 1162). Assim, os enfermeiros Especialistas em Saúde Materna, Obstetrícia e Ginecologia devem ser sensíveis às alterações biopsicossociais da menopausa e aos fatores de risco relacionados com o estilo de vida das mulheres, para que as possam ajudar, vigiar, objetivando a promoção da sua saúde e bem-estar, nesta fase da sua vida. A Ordem dos Enfermeiros (OE, 2011) reconhece o enfermeiro Especialistas em Saúde Materna, Obstetrícia e Ginecologia como o profissional que está habilitado para as consultas de enfermagem à mulher na menopausa, estando numa posição privilegiada para as informar, aconselhar, apoiar e vigiar a saúde das mulheres, contribuindo, deste modo, para uma transição positiva para a menopausa e uma vivência saudável, no seu todo, na pós-menopausa. Na categoria "Cuidados de enfermagem recebidos na menopausa" as subcategorias "“Esclarecimento de dúvidas", "Ajuda", "Capacitação da mulher para vivenciar a menopausa" e "Orientação e aconselhamento" foram as mais referenciadas pelas participantes, com nove e sete referências, respetivamente. As mulheres na menopausa têm várias necessidades de apoio. As principais necessidades devem ser compreendidas de acordo com a sua própria perspetiva, o que requer ajuda e esclarecimento de dúvidas para que possam suprir essas necessidades e preservar a sua qualidade de vida (Hoga, Rodolpho, Gonçalves \& Quirino, 2015, p. 279). Bernardes (2014) entrevistou oito mulheres na menopausa e constatou que várias condições que interferem no processo de transição para a menopausa acabam por ser sensíveis aos cuidados de enfermagem. Como tal, sugere que os enfermeiros desenvolvam cuidados antecipatórios, quer de forma individual, quer em grupo, prestando cuidados de enfermagem que capacitem as mulheres a vivenciar esta fase da sua vida. No seu estudo, a autora identificou como fatores, mais frequentes, que facilitam o processo de transição para a menopausa, a ocupação (profissional, familiar e tempos livres) e as pessoas que Ihes são significativas. Como fatores dificultadores, os mais referenciados pelas entrevistadas, foram a imprevisibilidade das alterações e a insatisfação com a orientação dos profissionais de saúde. É importante referir que, segundo Melleis, Sawyer, Messias \& Shumacher (2000, p. 13), os enfermeiros geralmente são os principais cuidadores de clientes e suas famílias em transição. Atendem às mudanças e necessidades que a transição acarreta para o quotidiano dos clientes e suas famílias. Além disso, os enfermeiros tendem a ser os cuidadores que preparam os clientes para impedir transições negativas e que facilitam o processo de aprendizagem de novas habilidades relacionadas com a saúde. Neste sentido, o enfermeiro Especialista em Saúde Materna, Obstetrícia e Ginecologia é o profissional com competências específicas para intervir no processo de transição e adaptação à menopausa, capacitando as mulheres para uma vivência mais positiva desta fase da sua vida. Em suma, a experiência da menopausa é influenciada por crenças e valores prevalentes no contexto sociocultural, a formação das mulheres e as formas como estas abordam as mudanças nessa fase da vida. Independentemente das circunstâncias envolvidas, as mulheres que vivenciam a menopausa precisam de ter as suas necessidades de cuidados e suporte correspondente identificados com base nas suas perspetivas pessoais e contextuais. 


\section{CONCLUSÕES}

Durante este período de transição da vida da mulher, que é a menopausa, são muitas as dúvidas que surgem acerca das transformações com as quais se deparam e com as quais tiveram que lidar.

Os resultados revelam que parte das mulheres entrevistadas encararam a menopausa de forma positiva. Porém, para outras, a menopausa teve um significado diferente, como uma fase de desconfortos, com alterações das condições vaginais, emocionais, orgânicas e alterações da atividade sexual, tendo havido quem procurasse ajuda profissional para fazer face a esses constrangimentos. As mulheres consideram, sobretudo, que os cuidados de enfermagem corresponderam às suas expetativas, uma vez que houve esclarecimento de dúvidas e que foram capacitadas para vivenciar a menopausa de forma positiva e saudável

Por conseguinte, importa reforçar que dotar as mulheres de literacia sobre a menopausa poderá ser uma estratégia para a redução da ansiedade e sentimentos negativos, tão comuns durante este período da sua vida. Assim, cabe ao enfermeiro Especialista em Saúde Materna, Obstetrícia e Ginecologia facultar às mulheres informações para que as possa preparar melhor, dar uma assistência humanizada, fazer uso da escuta ativa, dar apoio psicológico, ou seja, prestar cuidados holísticos à mulher, para que se possam minimizar as vivências negativas da menopausa e reduzir o desfasamento entre o que esperam dos cuidados que thes são prestados e a realidade que encontram.

Desenvolvemos, com a realização deste trabalho, uma consciência individual para o fenómeno em estudo. Deste modo, pretende desenvolver-se, na futura prática profissional, ações de formação em serviço, capacitando os enfermeiros especialistas em enfermagem de saúde materna e obstétrica para o cuidar mulheres a vivenciar este fenómeno, indo ao encontro das suas expetativas e promovendo experiências menos constrangedoras por parte das mulheres.

\section{FINANCIAMENTO E AGRADECIMENTOS}

Este trabalho é financiado por Fundos Nacionais através da FCT - Fundação para a Ciência e a Tecnologia, I.P., no âmbito do projeto Refª UIDB/00742/2020. Agradecemos adicionalmente ao Politécnico de Viseu pelo apoio prestado, UICISA:E; PV+Inclusão; SPECULA, CLAIM Politécnico de Viseu; Rede de Ensino Superior em Mediação Intercultural e Programa Mentores para Migrantes do Alto Comissariado para as Migrações; e Capítulo Phi Xi da Sigma Theta Tau International.

\section{REFERÊNCIAS BIBLIOGRÁFICAS}

Beck, V., Brewis, J., \& Davies, A. (2019). Women's experiences of menopause at work and performance management. Organization. Doi:10.1177/1350508419883386

Bernardes, M. (2014). Transição para a menopausa: das condições aos fatores sensiveis aos cuidados de enfermagem. (Dissertação de Mestrado). Escola Superior de Enfermagem de Coimbra. Coimbra. Acedido em http://repositorio.esenfc.pt/?url=11dXABiQ

Bogdan, R. C., \& Biklen, S. K. (2007). Chapter 4: Qualitative Data. Qualitative research for education: An introduction to theories and methods (5th ed.) (pp. 133-142 ONLY). Boston: Allyn and Bacon.

Chae, H.D., Choi, S.Y., Cho, E.J., Cho, Y.M., Lee, S.R., Lee, E.S. et al. (2014). Awareness and experience of menopausal symptom and hormone therapy in korean postmenopausal women. J Menopausal Med.; 20, 713. doi: $10.6118 / \mathrm{jmm} \cdot 2014.20 .1 .7$.

Dicionário Etimológico. Menopausa. Origem da palavra menopausa. Acedido em https://www.dicionarioetimologico.com.br/menopausa/

Dillaway, H. (2020). Living in Uncertain Times: Experiences of Menopause and Reproductive Aging (Capítulo 21). C. Bobel et al. (eds.). The Palgrave Handbook of Critical Menstruation Studies (pp. 253-268). https://doi.org/10.1007/978-981-15-0614-7_21 
Gass, M.L., Cochrane, B.B., Larson, J.C. et al. (2011). Patterns and predictors of sexual activity among women in the Hormone Therapy trials of the Women's Health Initiative. Menopause; 18(11), 1160-1171. doi: 10.1097/gme.0b013e3182227ebd.Acedido em https://www.ncbi.nlm.nih.gov/pubmed/21983008

Gault, A.M. (2015). Health by numbers: Menopause. Quality Health Medical Ctr. Acedido em http://www.qualityhealth.com/menopause-articles/health-numbers-menopause

Hoga, L., Rodolpho, J., Gonçalves, B. \& Quirino, B. (2015). Women`s experience of menopause: a systematic review of qualitative evidence. JBI Database of Systematic Reviews and Implementation Reports; Vol. 13(8), 250-337. Doi: 10.11124/jbisrir-2015-1948.

Kaunitz, A. M., \& Manson, J. E. (2015). Management of Menopausal Symptoms. Obstetrics and gynecology, 126(4), 859-876. https://doi.org/10.1097/A0G.0000000000001058

Kingsberg, S.A., Wysocki, S., Magnus, L.. et al. (2013). Vulvar and vaginal atrophy in postmenopausal women: findings from the REVIVE (REal Women's Vlews of Treatment Options for Menopausal Vaginal ChangEs) survey. J Sex Med; 10, 1790-1799.

Meleis, A. I., Sawyer, L. M., Im, E. 0., Messias, D. K. H. \& Shumacher, K. (2000). Experience transitions: An emerging middle-range theory. Advances in Nursing Science; 23 (1), 12-28. Doi: 10.1097/00012272200009000-00006.

Nappi, R.E., \& Kokot-Kierepa, M. (2010). Women's voices in the menopause: results from an international survey on vaginal atrophy. Maturitas; 67, 233-238. Acedido em https://www.ncbi.nlm.nih.gov/pubmed/20828948

Pedras, C.R.N. (2017). Transição para a menopausa: necessidades e expectativas. (Dissertação de Mestrado). Escola Superior de Saúde de Enfermagem de Lisboa. Acedido em https://comum.rcaap.pt/bitstream/10400.26/20974/1/Disserta\%C3\%A7\%C3\%A30\%20C\%C3\%A9lia\%20 Pedras.pdf

Probo, A., Soares, N., Silva, V., \& Cabral, P. (2016). Níveis dos sintomas climatéricos em mulheres fisicamente ativas e insuficientemente ativas. Revista Brasileira de Atividade Física \& Saúde; 21(3), 246-254. https://doi.org/10.12820/rbats.v.21n3p246-254

Queiróz, A.A., Meireles, M.A., \& Cunha, S.R. (2007). Investigar para compreender. Loures: Lusociência, 2007. XI. ISBN 978-972- 8930-38-7.

Samarasiri, D.G.C.N., Rajanayake, I.T., Wattegedara, P.K., Kodituwakku, R.D., Senadheera, A.S.P.L. \& Silva, B.S.S. de (2017). Women's Experiences on Menopause: A Phenomenological Study. OUSL Journal; Vol. 12, 2, 4362. D0I: http://doi.org/10.4038/ouslj.v12i2.7407

Samarasiri, D.G.C.N., Rajanayake, I.T., Wattegedara, P.K., Kodituwakku, R.D., Senadheera, A.S.P.L. \& Silva, B.S.S. de (2017). Women's Experiences on Menopause: A Phenomenological Study. OUSL Journal; Vol. 12, 2, $43-$ 62. D0l: http://doi.org/10.4038/ouslj.v12i2.7407

Silva, L.D.C., \& Mamede, M.V. (2017). Desvelando os sentidos e significados do climatério em mulheres coronarianas. Cienc Cuid Saud; 16(2), 2-8. Doi: 10.4025/cienccuidsaude.v16i2.31719.

The North American Menopause Society. (2014). Clinical Care Recommendations - Chapter 1: Menopause. Acedido em http://www.menopause.org/publications/clinical-care-recommendations/chapter-1- menopause

Vale, E.G., \& Pagliuca, L.M.F. (2011) Construção de um conceito de cuidado de enfermagem: Contribuição para 0 ensino de graduação. Revista Brasileira de Enfermagem; 64, 106-113. http://dx.doi.org/10.1590/S0034-71672011000100016

Van Manen, M. (2017). But is it phenomenology? Qualitative Health Research, 27(6), 775-779. Acedido em https://doi.org/10.1177/1049732317699570

Waheed, K., Khanum, A., Ejaz, S., Butt, A.F., \& Randhawa, A. (2016). Quality of Life after Menopause in Pakistani Women. Gynecol Obstet (Sunnyvale); 6, 4, 1-3. D0l; 10.4172/2161-0932.1000367.

Yazdkhasti, M., Simbar, M., \& Abdi, F. (2015). Empowerment and Coping Strategies in Menopause Women: A Review. Iran Red Crescent Med J.; 17(3): e18944, 2-5. Doi: 10.5812/ircmj.18944 